\title{
Multiple brown tumours of tertiary hyperparathyroidism in a renal transplant recipient: A case report
}

\author{
Diego-Peres Magalhães ${ }^{1}$, Rafael-Lima-Verde Osterne ${ }^{1}$, Ana-Paula-Negreiros-Nunes Alves ${ }^{2}$, Paulo-Sergio-da \\ Silva Santos ${ }^{3}$, Roberto-Brasil Lima ${ }^{4}$, Fabrício-Bitu Sousa ${ }^{2}$
}

\footnotetext{
${ }^{1}$ MSc, Post-graduate program in Dentistry at the Federal University of Ceará in Ceará, Brazil

${ }^{2}$ PHD, Associate Professor of the Department of Odontology at the Federal University of Ceará, Brazil

${ }^{3}$ DDS in Oral Pathology - FOUSP, Member of the Hospital Santa Isabel Staff, São Paulo, Brazil

${ }^{4}$ MS, School of Medicine Sciences of Santa Casa of São Paulo, São Paulo, Brazil
}

Correspondence:

Tibúrcio Cavalcante St, 1222, apt501

Aldeota-Zip code: 60125100

Fortaleza/CE, Brazil,

diegopmagalhaes@hotmail.com

Received: 28/03/2009

Accepted: 02/08/2009
Magalhães DP, Osterne RLV, Alves APNN, Santos PSD, Lima RB, Sousa FB. Multiple brown tumours of tertiary hyperparathyroidism in a renal transplant recipient: A case report. Med Oral Patol Oral Cir Bucal. 2010 Jan 1;15 (1):e10-3.

http://www.medicinaoral.com/medoralfree01/v15i1/medoralv15i1p10.pdf

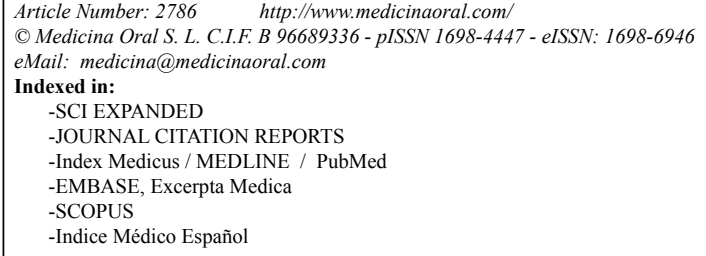

\begin{abstract}
Tertiary Hyperparathyroidism (HPT) is an uncommon condition that affects patients with secondary HPT after a successful kidney transplant. Bone alterations are the main consequences of this endocrine condition, including the development of an osteolytic lesion called brown tumour. This paper reports an unusual case of multiple brown tumours located in the maxilla and mandible in a 58-year-old renal transplant recipient, with tertiary HPT. Incisional biopsies were performed and, in both samples, the histopathological diagnoses were giant cell lesions. Due to these diagnoses, the medical history of chronic renal failure, and high levels of serum calcium and PTH, lesions were diagnosed as multiple brown tumours of tertiary HPT. The lesions showed regression within the two months following a total parathyroidectomy. Minimal local interventions were established to make an early diagnosis and to treat these lesions. The diagnosis of primary oral lesions was very important in order to demonstrate the relevance of interdisciplinary activity and the inclusion of dentists as part of organ transplant units.
\end{abstract}

Key words: Brown tumor, tertiary hyperparathyroidism, oral cavity, renal transplant recipient. 


\section{Introduction}

Hyperparathyroidism (HPT) is an endocrine condition that occurs in three categories: primary, secondary and tertiary. Primary HPT occurs with a hyperfunction of one or more parathyroids, causing an increase of parathyroid hormone (PTH) secretion and resulting in hypercalcemia. Secondary HPT, normally related to chronic renal failure, occurs with a decrease of vitamin D production or with hypocalcemia, making the glands to produce a high quantity of PTH. Tertiary HPT can occur in a small number of patients, when the parathyroids activity turns autonomous and excessive, leading to hypercalcemia (1). Bone alterations are the main consequences of this endocrine condition, including the development of an osteolitic lesion called brown tumor $(2,3)$.

This paper reports a case of multiple brown tumours located in maxilla and mandible in a renal transplant recipient with tertiary HPT. The lesions showed regression after a total parathyroidectomy.

\section{Case Report}

A 58-year-old woman was referred to Study Nucleus in Special Care Dentistry (NEPE), Federal University of Ceará, Brazil, in November 2007, with the complain of pain in the lower right third molar (tooth 48). Medical history was significant for chronic renal failure, and the patient underwent hemodialysis since 1999. In 2004, she was submitted to a renal transplant, but in 2006 the organ was rejected, and a new transplant surgery was performed. In this period, she developed diabetes mellitus due to the immunosuppressive therapy.

Physical extra oral examination was unremarkable, and facial deformity was not observed. Physical intraoral examination showed an extend amalgam restoration and gingival tissue covering the crown of tooth 48 . Pericoronitis signs were observed, and the tooth showed intense mobility and pain. Moreover, a nodular lesion, with $5 \mathrm{~mm}$ approximately, and coloration similar to normal mucosa, were detected in the upper left gum, mesially to the upper left second molar (tooth 27).

Radiographic exam showed a non-defined radiolucent area, with irregular contours located in the tooth 48 region, extended to the left mandible ramus. Radiolucent areas were also observed on the distal roots of the lower left first molar and lower right second molar (teeth 36 and 47). In maxilla, a well-defined radiolucent region was observed between the upper left second premolar and upper left second molar (teeth 25 and 27). Radiographic characteristics of renal osteodystrophy, such as "unpolished glass" aspect of the trabecular bone and obliteration of the root channels, were observed (Fig.1). Pulp vitality tests were performed in the teeth with radiolucent areas, being all teeth with vitality. Based on the clinical-radiographic findings, a decision was made to the complete removal of tooth 48 because of pain and infection signs, which could increase due to the immunosupression therapy. Incisional biopsies were indicated to the lesion related to tooth 48 and the nodular lesion in maxilla. Hematologic exams, such as hemogram, coagulation testing, and glycosylated hemoglobin were performed, with no alterations that could counter indicate the surgical procedure. The removal of the tooth 48 was performed, and pathologic tissue was collected through the dental alveolus, and from the nodular maxillary lesion.

In both samples, the histopathological exams revealed multinucleated osteoclast-like giant cells in a colagenous stroma, with oval and fusiforms cells in the background. The diagnoses were giant cell lesions for both lesions (Fig. 2). Due to these diagnoses and the medical history of chronic renal failure, laboratorial exams were

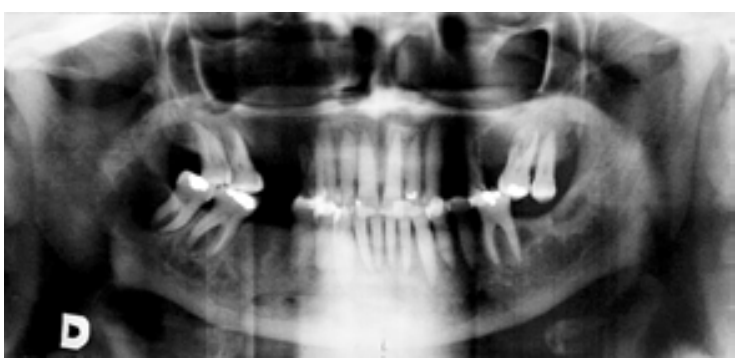

Fig. 1. Orthopantogram with radiolucent areas in maxilla and mandible.

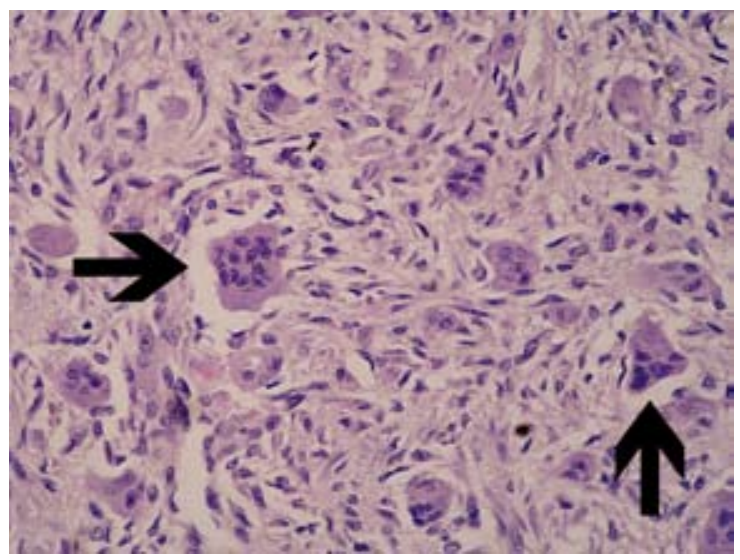

Fig. 2. Multinucleated osteoclast-like giant cells (Hematoxylin and eosin stain - 40x).

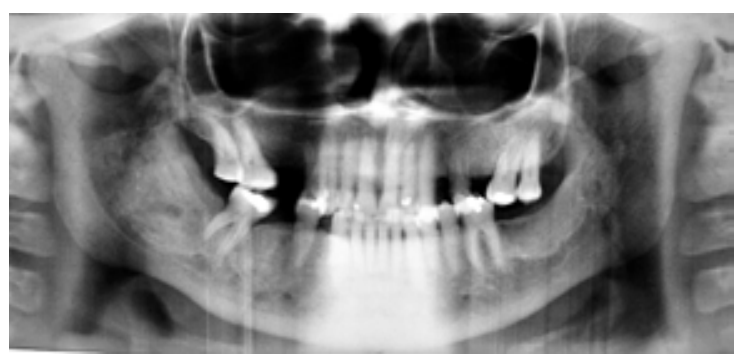

Fig. 3. Orthopantogram showing healing of the osteolitic lesions. 
performed to search possible hormonal changes related to the parathyroids. The results were: 15,8 [normal 8,6 to 10,2$] \mathrm{mg} / \mathrm{dL}$ for serum calcium; $2,8(2,7$ to 4,5$) \mathrm{mg} /$ $\mathrm{dL}$ for serum phosphorous; and 1090,0 (10 to 65) pg/mL for serum PTH. Because these findings indicated that both the maxilla and mandible were affected by giant cell lesions associated high levels of serum calcium and PTH after renal transplant surgery, the lesions were diagnosed as multiples brown tumours of tertiary HPT. The patient was referred to the Nephrology service of the Walter Cantídio University Hospital, Ceará Federal University, Brazil. No osteolitic lesions were found on the other bones, and a total parathyroidectomy was performed in July 2008 to control high levels of PTH and to resolve the radiolucent lesions of the jaws. In September 2008 , the patient showed adequate cicatrization of the oral mucosa, and radiographic exams demonstrated the presence of osseous healing in areas that had previous had osteolitic lesions in maxilla and mandible (Fig.3).

\section{Discussion}

Tertiary HPT is an uncommon condition, affecting up to $8 \%$ of patients with secondary HPT after a successful renal transplant (2). High quantities of PTH secreted by the parathyroid glands in this condition results in a serum hypercalcemia (4).

Bone lesions are one of the main manifestations of HPT. They can alternate from a generalized osteopenia, cystic lesions, and bone resorptions, leading to brown tumors (5). The ribs, clavicles, pelvic girdle, and the mandible are the most often involved bones (6).

The brown tumor presents itself as a friable redbrowned mass, representing the final stage of HPT (7). It is an osteolitic lesion, developed due to changes in bone metabolism caused by high serum concentration of PTH. Mandible involvement is common, showing solitary or multiple lesions. Osteolitic areas in maxilla are considered rare. Radiographically, lesions are characterized as well-defined radiolucent areas, unilocular or multilocular. Long-term injures commonly produce a significant expansion of cortical (8). Characteristic radiographic findings of HPT can also be found in patients with brown tumors, such as a widespread loss of the lamina dura, and changes in the pattern of trabecular bone of the jaws. When it occurs in its peripheral variant, bone surface erosion is detected in the affected area (9). Based on those radiographic characteristics, the maxillary lesion presented in this case was diagnosed as a central tumor brown.

Histologically, brown tumor is characterized by an abundant stroma, consisting of bundles of spindle or oval cells, and several multinucleated osteoclast-like giant cells. Calcified material can be found, as well as areas with extravasation of red blood cells and pigmentation by haemosiderin (10). These findings are not pathognomonic, making it necessary to perform a differential diagnosis with other lesions such as Aneurysmal Bone Cyst, Cherubism and Central Giant Cell Granuloma (11). Clinical and endocrine status of the patient is indispensable to make the differential diagnosis of the Brown Tumor.

Total parathyroidectomy is indicated to treat tertiary HPT, restoring normal serum PTH values, and increasing bone mineral density (12). Patients who underwent partial removal of the glands are more likely to remain in HPT (13). Normal rates of PTH restore the normal metabolism of calcium, causing the osteolytic lesions of the brown tumor to regress (14). Surgical intervention, by curettage and excision of the lesion, is indicated if bone healing does not occur. In the present report, only total parathyroidectomy was required to heal the affected bone areas, a fact that occurred in just two months after the excision of the glands. In aggressive lesions, conservative therapies are reported in the literature, such as the use of intralesional corticosteroids and calcitonin, requiring only a possible osteoplasty after the recalcification of the osteolytic sites (15).

This case demonstrates a direct effect of excessive production of PTH after renal transplantation, and its direct implications in the maxillofacial complex. The presence of multiple lesions, including including lesions in the region of the maxilla, together with the rapid healing of osteolytic lesions in just two months after total parathyroidectomy, makes the case unusual.

In conclusion, bone alterations are the main consequences of tertiary HPT, including the development of brown tumors. In this case, the diagnosis of primary oral lesions was very important to demonstrate the relevance of interdisciplinary activity and the insertion of dentists in organ transplantation staffs. Minimal local interventions were established to make an early diagnosis and to treat these lesions.

\section{References}

1. Ahmad R, Hammond JM. Primary, secondary, and tertiary hyperparathyroidism. Otolaryngol Clin North Am. 2004;37:701-13.

2. Nieto J, Ruiz-Cuevas P, Escuder A, Regas J, Callis L. Tertiary hyperparathyroidism after renal transplantation. Pediatr Nephrol. 1997;11:65-8.

3. Fernández-Sanromán J, Antón-Badiola IM, Costas-López A. Brown tumor of the mandible as first manifestation of primary hyperparathyroidism: diagnosis and treatment. Med Oral Patol Oral Cir Bucal. 2005;10:169-72.

4. Gasparri G, Camandona M, Abbona GC, Papotti M, Jeantet A, Radice E, et al. Secondary and tertiary hyperparathyroidism: causes of recurrent disease after 446 parathyroidectomies. Ann Surg. 2001;233:65-9.

5. De Francisco AL. Secondary hyperparathyroidism: review of the disease and its treatment. Clin Ther. 2004;26:1976-93.

6. Keyser JS, Postma GN. Brown tumor of the mandible. Am J Otolaryngol. 1996;17:407-10.

7. Triantafillidou K, Zouloumis L, Karakinaris G, Kalimeras E, Iordanidis F. Brown tumors of the jaws associated with primary or secondary hyperparathyroidism. A clinical study and review of the literature. Am J Otolaryngol. 2006;27:281-6. 
8. Adachi PL, Da Silva Santos PS, De Magalhães MH, Martins MT. Renal osteodystrophy manifesting as localized enlargement of the jaw. Nephrol Dial Transplant. 2007;22:2398-9.

9. Guimaraes ALS, Marques-Silva L, Gomes CC, Castro WH, Mesquita RA, Gomez RS. Peripheral brown tumour of hyperparathyroidism in the oral cavity. Oral Oncol Extra. 2006;3:91-3.

10. Pérez-Guillermo M, Acosta-Ortega J, García-Solano J, RamosFreixá J. Cytologic aspect of brown tumor of hyperparathyroidism. Report of a case affecting the hard palate. Diagn Cytopathol. 2006;34:291-4.

11. Selden HS. Central giant cell granuloma: a troublesome lesion. J Endod. 2000;26:371-3.

12. Collaud S, Staub-Zähner T, Trombetti A, Clerici T, Marangon $\mathrm{N}$, Binet I, et al. Increase in bone mineral density after successful parathyroidectomy for tertiary hyperparathyroidism after renal transplantation. World J Surg. 2008;32:1795-801.

13. Kebebew E, Duh QY, Clark OH. Tertiary hyperparathyroidism: histologic patterns of disease and results of parathyroidectomy. Arch Surg. 2004;139:974-7.

14. Loushine RJ, Weller RN, Kimbrough WF, Liewehr FR. Secondary hyperparathyroidism: a case report. J Endod. 2003;29:272-4.

15. Pinto LP, Cherubinim K, Salum FG, Yurgel LS, De Figueiredo MA. Highly aggressive brown tumor in the jaw associated with tertiary hyperparathyroidism. Pediatr Dent. 2006;28:543-6. 